Results The FTA-ABS was used as the reference since it has been considered the Gold Standard" among treponemal tests. The analytical sensitivity of the six tests fell into three statistically different groups (from lowest to highest): (1) the FTA-ABS, the TP-PA, and the TrepChek, (2) the LIAISON and the TrepID, and (3) the TrepSure. In addition, there were 24 sera that were found nonreactive by FTA-ABS and nine found non-reactive by TrepChek that were reactive by at least two other treponemal tests.

Conclusions These results highlight significant differences in the analytical sensitivity of various treponemal tests and could explain some discordant results between treponemal tests used to confirm screening EIAs and CIAs. First, the FTA-ABS should no longer be considered the "Gold Standard". Second, low titre sera could appear non-reactive if relatively insensitive tests are used for the either screening or confirmatory tests. Third, the question arises, "Should initial screening tests be confirmed by treponemal tests with lower analytical sensitivity?" Future patient studies could verify these results, so that a more accurate standard protocol for syphilis testing can be established.

\section{P3-S6.06 DOES THE CONCEPT OF EARLY AND LATE LATENT SYPHILIS HOLD WELL IN TODAY'S SCENARIO?}

doi:10.1136/sextrans-2011-050108.480

D De, A Kanwar. Postgraduate Institute of Medical Education and Research, Chandigarh, India

Background Latent syphilis refers to the asymptomatic stage in the natural evolution of syphilis in a patient not treated or inadequately/inappropriately treated for syphilis. Latent syphilis has been categorised as early latent and late latent for therapeutic purposes. Indiscriminate and inadequate over the counter use of antibiotics (happenstance) and HIV co-infection in present day scenario tends to change natural evolution of syphilis. Even though some patients remain asymptomatic without any historical evidence of clinical manifestation of syphilis, serologically they continue to reflect the ongoing infection process; the so called syphilis of unknown duration.

Objective To determine the sero-epidemiological characteristics of patients with syphilis of unknown duration registered to a sexually transmitted disease (STD) clinic in an Institute setting.

Patients and Methods Clinic records of patients registered to the STD clinic between 2006 and 2010 were retrieved. For study purposes, syphilis of unknown duration was defined as positivity with Treponema Pallidum Haemagglutination assay (TPHA) irrespective of Venereal Disease Research Laboratory (VDRL) titre in patients who did not have any clinical sign of syphilis including neurological and cardiac, during clinic entry; had no features to suggest syphilis in the past and had not been treated with parenteral penicillin.

Results Of the 76 patients registered during the study period, 52 $(68 \%)$ satisfied the diagnosis of syphilis of unknown duration. Age of patients was between 20 and 56 years (mean 29.76, standard deviation 8.32 ). Males outnumbered females by a ratio of $2.25: 1$. Majority $(33,63.4 \%)$ of the patients were direct walk-in with positive serology results done in private laboratories, mostly in patients who are habituated to unprotected extramarital sexual intercourse. Others were referred from obstetric clinics (15.4\%), private practitioners (15.4\%), HIV clinic (1.9\%), transfusion medicine $(1.9 \%)$ and urology clinic (1.9\%). Majority patients $(31,59.61 \%)$ showed low titre VDRL (upto $1: 8)$ positivity while $5(9.6 \%)$ were VDRL non-reactive.

Conclusion In majority of syphilis patients, duration of latency could not be determined due to lack of history of clinical lesions of early syphilis or reliable history of time of acquisition of infection. Consequently, the categorisation of latent syphilis to early and late phases in present day scenario appears redundant.

\section{P3-S6.07 ABSTRACT WITHDRAWN}

\section{P3-S6.08 DETECTION OF THE 23S RRNA POINT MUTATIONS (A2058G AND A2059G) ASSOCIATED WITH AZITHROMYCIN RESISTANCE IN TREPONEMA PALLIDUM USING A TAQMAN-BASED REAL-TIME TRIPLEX-PCR ASSAY}

doi:10.1136/sextrans-2011-050108.482

C Y Chen, K H Chi, A Pillay, E Nachamkin, R Ballard. CDC, Atlanta, USA

Background To develop a TaqMan-based real-time allelic discrimination assay for the simultaneous detection of two point mutations (A2058G and A2059G) in the 23S rRNA gene of T pallidum that have been associated with azithromycin treatment failures.

Methods Initially, two TaqMan-based real-time duplex PCR assays were used to detect the A2058G and A2059G point mutations within the $23 \mathrm{~S}$ rRNA gene of $T$ pallidum. Genotyping results from these assays were then compared to a previously described real-time PCR assay using fluorescence resonance energy transfer (FRET) probes and melting curve analysis that is specific for the detection of the A2058G point mutation. Subsequently, a real-time triplex PCR was developed to distinguish the A2058G from the A2059G point mutation in a single assay and the results were confirmed by pyrosequencing.

Results Sixteen of 67 (23.9\%) T pallidum-positive specimens collected from patients with genital ulcer disease in the US were found to have the A2059G point mutation. These strains were previously characterised as having azithromycin-susceptible genotypes (no point mutations in the 23S rRNA gene). The A2059G mutation was confirmed by a real-time duplex PCR assay containing the TaqMan probe specific for the mutation and by pyrosequencing. None of the $T$ pallidum strains examined to date had both point mutations. The real-time triplex PCR assay was able to differentiate azithromycin-susceptible genotypes from resistant genotypes containing either the A2058G or A2059G point mutation in a single assay. The limit of detection of the assay was $1-10$ copies using $23 \mathrm{~S}$ rRNA gene fragments that were cloned into a plasmid.

Conclusions The previously reported real-time PCR detection platforms were designed to detect only the A2058G point mutation and were unable to differentiate $T$ pallidum strains with susceptible genotypes from resistant genotypes with the A2059G mutation. The new TaqMan-based real-time allelic discrimination assay offers an alternative to the previously described PCR/RFLP method to rapidly detect both point mutations associated with azithromycin resistance in $T$ pallidum. The prevalence of $\mathrm{T}$. pallidum strains that harbour point mutations in the $23 \mathrm{~S}$ rRNA gene associated with resistance to azithromycin might be much higher than previously estimated.

\section{P3-S6.09 ABSTRACT WITHDRAWN}

\section{P3-S6.10 ANALYSIS OF SYSTEMIC AND CUTANEOUS IMMUNE RESPONSES HELPS EXPLAIN THE DUALITY OF IMMUNE EVASION AND RECOGNITION IN SECONDARY SYPHILIS}

doi:10.1136/sextrans-2011-050108.483

A Cruz, L Ramirez. CIDEIM, CALI, Colombia

Background Despite the robust adaptive immune responses associated with secondary syphilis (SS), which include the presence of high titres of anti-T pallidum (Tp) antibodies with opsonizing 